\title{
Processing and validation of JEFF-3.1.1 and ENDF/B-VII.0 group-wise cross section libraries for shielding calculations
}

\author{
M. Pescarini ${ }^{1}$, V. Sinitsa ${ }^{2}$, R. Orsi ${ }^{1}$ and M. Frisoni ${ }^{1}$ \\ ${ }^{1}$ ENEA, CR “E. Clementel”, Via Martiri di Monte Sole 4, 40129 Bologna, Italy \\ ${ }^{2}$ NRC "Kurchatov Institute”, 1, Academica Kurchatova pl., 123182 Moscow, Russia
}

\begin{abstract}
This paper presents a synthesis of the ENEA-Bologna Nuclear Data Group programme dedicated to generate and validate group-wise cross section libraries for shielding and radiation damage deterministic calculations in nuclear fission reactors, following the data processing methodology recommended in the ANSI/ANS-6.1.2-1999 (R2009) American Standard. The VITJEFF311.BOLIB and VITENDF70.BOLIB finegroup coupled $n-\gamma(199 n+42 \gamma$ - VITAMIN-B6 structure) multi-purpose cross section libraries, based on the Bondarenko method for neutron resonance self-shielding and respectively on JEFF-3.1.1 and ENDF/B-VII.0 evaluated nuclear data, were produced in AMPX format using the NJOY-99.259 and the ENEA-Bologna 2007 Revision of the SCAMPI nuclear data processing systems. Two derived broad-group coupled $n-\gamma(47 n$ $+20 \gamma$ - BUGLE-96 structure) working cross section libraries in FIDO-ANISN format for LWR shielding and pressure vessel dosimetry calculations, named BUGJEFF311.BOLIB and BUGENDF70.BOLIB, were generated by the revised version of SCAMPI, through problem-dependent cross section collapsing and self-shielding from the cited fine-group libraries. The validation results on the criticality safety benchmark experiments for the fine-group libraries and the preliminary validation results for the broad-group working libraries on the PCA-Replica and VENUS-3 engineering neutron shielding benchmark experiments are reported in synthesis.
\end{abstract}

\section{Introduction}

An increasing interest in the generation and testing of updated group-wise cross section libraries for reactor shielding applications is presently justified by a specific evolution of the use, in the last two decades, of the three-dimensional (3D) deterministic radiation transport codes like, e.g., the discrete ordinates $\left(\mathrm{S}_{\mathrm{N}}\right)$ code TORT [1]. In fact, if supported by pre/post-processor ancillary systems of codes (e.g., BOT3P [2] [3], TORTWARE [4], etc.), dedicated to the automatic mesh grid generation of the spatial model together with its graphical verification, the 3D deterministic codes become extremely competitive, also in the description of complex geometries, in comparison with the stochastic codes (e.g., the Monte Carlo code MCNP). Concerning the pre/post-processor systems, it is underlined that the ENEA-Bologna Nuclear Data Group developed the BOT3P-5.3 system, presently freely distributed by OECD-NEADB and ORNL-RSICC. BOT3P is based on combinatorial geometry algorithms and, although initially dedicated to the DORT 2D and TORT 3D $\mathrm{S}_{\mathrm{N}}$ codes of the DOORS [5] system, it can now easily generate detailed spatial mesh grids also for any other possible transport code through simple interfaces dedicated to manage the BOT3P binary output files. The 
generation methodology of the modern group-wise cross section libraries for fission reactor shielding applications must be performed in two steps, as reported in the ANSI/ANS-6.1.2-1999 (R2009) [6] American National Standard "Neutron and Gamma-Ray Cross Sections for Nuclear Radiation Protection Calculations for Nuclear Power Plants", reapproved in 2009. Moreover this standard explicitly recommends the ORNL DLC-0185/VITAMIN-B6 [7] and DLC-0184/BUGLE-96 [7] group-wise cross section libraries as reference tools for the cited applications. It is stated in particular that it is necessary 1) to process the evaluated data into fine-group (hundreds of groups), pseudoproblem-independent libraries based on the Bondarenko [8] neutron resonance self-shielding factor method and 2) to obtain from these libraries broad-group (tenths of groups) working libraries, generated through fine-group cross section collapsing with pre-calculated problem-dependent neutron and photon weighting spectra, including parameterized sets of problem-dependent selfshielded cross sections for the specific applications. In this paper it is synthetically described the ENEA-Bologna Nuclear Data Group activity, developed since 2009, addressed to generate and to validate fine-group pseudo-problem-independent libraries and derived broad-group working libraries for LWR shielding and radiation damage, based on the OECD-NEADB JEFF-3.1.1 [9] [10] and US ENDF/B-VII.0 [11] evaluated data libraries.

\section{Processing and validation of fine-group cross section libraries}

The NEA-1869/VITJEFF311.BOLIB [12] (see Figure 1) and NEA-1870/VITENDF70.BOLIB [13] fine-group coupled $n-\gamma$ cross section libraries were processed with an automatized and standardized calculation chain including the two following nuclear data processing systems: NJOY-99.259 [14] (updating "upnea049") and "ENEA-Bologna 2007 Revision [15] of SCAMPI [16]". VITJEFF311.BOLIB and VITENDF70.BOLIB adopt the same neutron and photon energy group structures (199 $\mathrm{n}+42 \gamma)$ as the VITAMIN-B6 (ENDF/B-VI.3) library with the 199 neutron energy groups in the energy range included within $1.0 \mathrm{E}-05 \mathrm{eV}$ and $1.9640 \mathrm{E}+07 \mathrm{eV}$ and the 42 photon energy groups included in the energy range within $1.0 \mathrm{E}+03 \mathrm{eV}$ and $3.0 \mathrm{E}+07 \mathrm{eV}$. The thermal neutron energy range, where the upscatter is possible, contains 36 groups and has $5.043 \mathrm{eV}$ as the uppermost boundary. VITJEFF311.BOLIB and VITENDF70.BOLIB are pseudo-problemindependent libraries based on the Bondarenko (f-factor) method for the treatment of neutron resonance self-shielding and temperature effects and contain respectively 176 and 177 standard (not bound) nuclide data files processed at 4 temperatures $\left(300^{\circ} \mathrm{K}, 600{ }^{\circ} \mathrm{K}, 1000^{\circ} \mathrm{K}\right.$ and $\left.2100{ }^{\circ} \mathrm{K}\right)$. Most materials were processed with 6 to 8 values for the background cross section $\sigma_{0}$ with nearly all materials processed at the following values of $\sigma_{0}: 1,10,1.0 \mathrm{E}+2,1.0 \mathrm{E}+3,1.0 \mathrm{E}+4$ and $1.0 \mathrm{E}+10$ barns. In both libraries, differently from the ORNL VITAMIN-B6 and VITAMIN-B7 [17] (ENDF/B-VII.0) similar libraries, an additional $\sigma_{0}$ numerical value equal to 0.01 barns was introduced in the data processing of the Fe-56 data files in order to improve the self-shielding of Fe-56 in pure natural iron. Thermal scattering cross sections were processed at all the temperatures available in the JEFF-3.1.1 and ENDF/B-VII.0 thermal scattering law data files for 6 additional bound nuclides: $\mathrm{H}-1$ in light water, $\mathrm{H}-1$ in polyethylene, $\mathrm{H}-1$ in zirconium hydride, $\mathrm{H}-2$ in heavy water, $\mathrm{C}$ in graphite and $\mathrm{Be}$ in beryllium metal. Several modules of NJOY were used to process the neutron interaction, the photon production and the photon interaction data from the ENDF-6 format of the JEFF-3.1.1 and ENDF/BVII.0 evaluated data to the NJOY GENDF group-averaged format. In order to process the evaluated data files into the AMPX format, it was necessary to develop the updated and corrected version of SCAMPI previously cited, freely released to OECD-NEADB and ORNL-RSICC. In particular this version of SCAMPI is able to generate and to read data in AMPX format, through the revised and corrected SMILER module. Concerning the present work, the revised SMILER was employed 1) to read the double-precision GENDF binary files from NJOY, 2) to translate the GENDF NJOY file into the AMPX master format for the VITJEFF311.BOLIB and VITENDF70.BOLIB libraries and, finally, 3) to calculate the total (prompt + delayed) neutron fission spectra and the average numbers of neutrons emitted per fission of the fissionable nuclides. In parallel, automatic multiplication of the term of $\ell$-th order of the Legendre polynomial $\left(\mathrm{P}_{\ell}\right)$ expansion of the scattering cross section matrix 
by the $(2 \ell+1)$ factor was performed by SMILER for all the data files of the nuclides contained in the libraries. The maximum order of scattering for both neutrons and photons is $\mathrm{P}_{7}$, for nuclides with $Z=1$ through $Z=29$ (copper), and $P_{5}$ for the remainder of the nuclides. The neutron weighting function is of the form typically chosen for fission reactor shielding problems, i.e. it consists of a smoothly varying combination of a Maxwellian thermal spectrum, a fission spectrum and a " $1 / \mathrm{E}$ " slowing down spectrum. The breakpoint energies for this 3-region spectrum are similar to those used in the VITAMIN-C [18] library: between the Maxwellian and 1/E shapes the breakpoint energy is at 0.125 $\mathrm{eV}$ while between $1 / \mathrm{E}$ and fission spectrum shapes it is at $820.8 \mathrm{keV}$. The nuclear fission temperature value $(\theta=1.273 \mathrm{MeV})$ used in the data processing has been adjusted, with respect to the value $(\theta=1.41 \mathrm{MeV})$ adopted in VITAMIN-C, to better reflect the neutron spectrum in a thermal reactor. VITJEFF311.BOLIB and VITENDF70.BOLIB were extensively tested, through the XSDRNPM 1D transport module of SCAMPI in the $\mathrm{S}_{16}-\mathrm{P}_{5}$ approximation, on the same set of about 80 thermal, intermediate and fast neutron spectrum criticality safety benchmark experiments taken from the ICSBEP [19] Handbook. About $80 \%$ of the k-effective calculated results obtained with the two libraries are contained within uncertainties corresponding to two standard deviations $( \pm 2 \sigma)$ of the experimental or "benchmark model" k-effective values for which a normal distribution is assumed. The results obtained with VITENDF70.BOLIB appear definitely better in the U-233 fast benchmark experiments. From these fine-group libraries it is possible to generate, through the ENEA-Bologna 2007 Revision of SCAMPI, working libraries of collapsed and self-shielded cross sections in the AMPX or FIDO-ANISN formats.

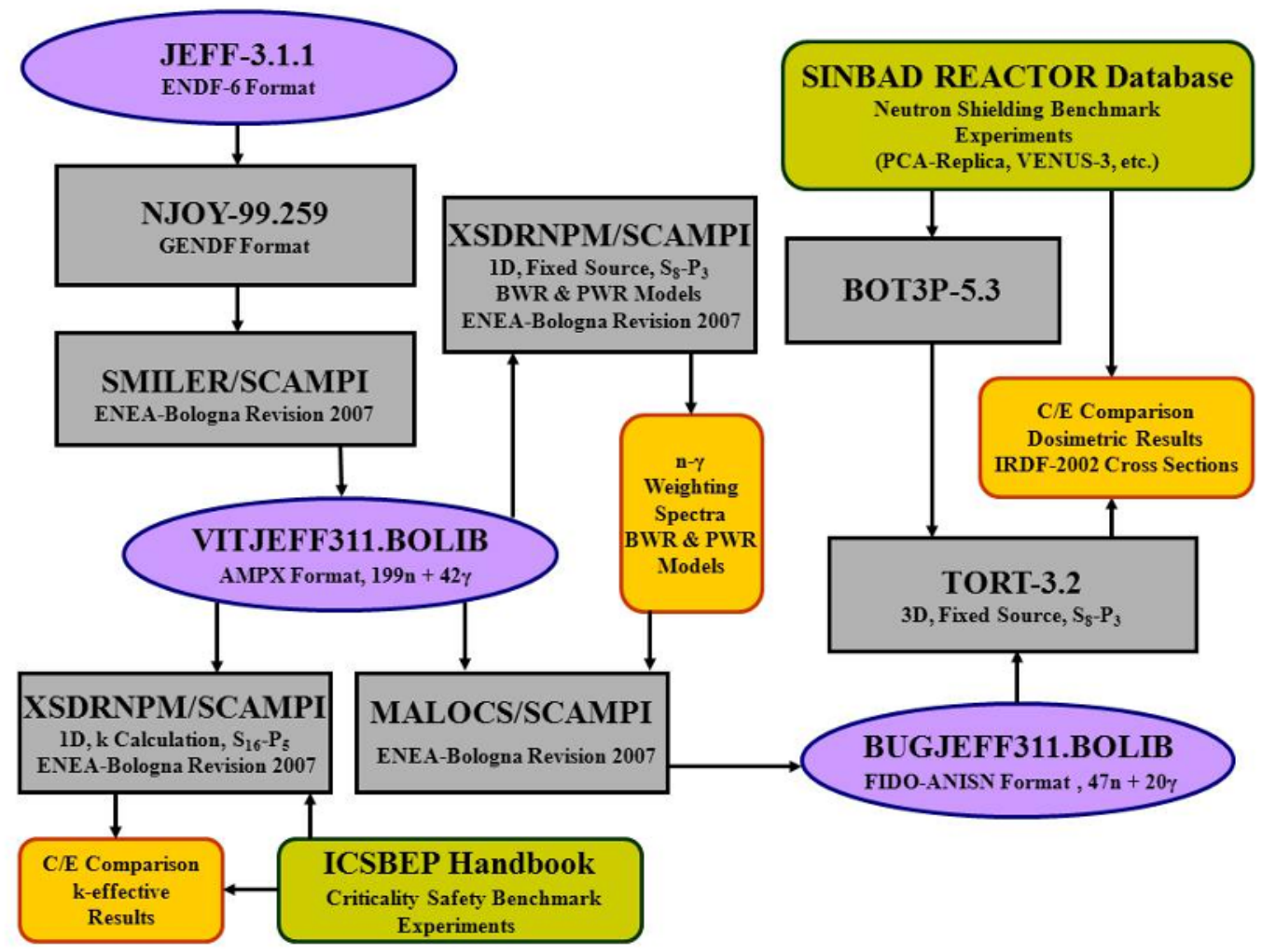

Fig. 1. Simplified data processing and validation flow-chart of the JEFF-3.1.1 libraries. 


\section{Processing and validation of broad-group cross section libraries}

Two broad-group coupled $n-\gamma$ working cross section libraries in FIDO-ANISN format, based on the JEFF-3.1.1 (see Figure 1) and the ENDF/B-VII.0 evaluated nuclear data libraries and named respectively NEA-1866/BUGJEFF311.BOLIB [20] and BUGENDF70.BOLIB [21], were generated through the ENEA-Bologna 2007 Revision of SCAMPI. These libraries have features similar to the BUGLE-96 (ENDF/B-VI.3) broad-group library and the same neutron and photon energy group structure $(47 \mathrm{n}+20 \gamma)$ with the 47 neutron energy groups covering the energy range $1.0 \mathrm{E}-05 \mathrm{eV}-$ $1.7332 \mathrm{E}+07 \mathrm{eV}$ and the 20 photon groups covering the energy range $1.0 \mathrm{E}+04-1.4 \mathrm{E}+07 \mathrm{eV}$. As previously performed at ORNL, where BUGLE-96 was obtained through proper cross section collapsing of VITAMIN-B6 through SCAMPI, the ENEA-Bologna BUGLE-type libraries were generated in a similar way. They were obtained through cross section collapsing from VITJEFF311.BOLIB and VITENDF70.BOLIB, strictly following the procedure recommended by the ANSI/ANS-6.1.2-1999 (R2009) standard. BUGJEFF311.BOLIB and BUGENDF70.BOLIB are singularly equipped with seven blocks of cross sections. The first one contains infinite dilution $\left(\sigma_{0}=\right.$ $1.0 \mathrm{E}+10$ barns) cross sections at the temperature of $300{ }^{\circ} \mathrm{K}$ degrees for all the materials (more than 180) contained respectively in VITJEFF311.BOLIB and VITENDF70.BOLIB: these cross sections were obtained through direct cross section collapsing of the fine-group VITJEFF311.BOLIB and VITENDF70.BOLIB corresponding cross sections, through the MALOCS module of SCAMPI, using a neutron and photon spectrum pre-calculated in a concrete PWR biological shield. The other six blocks contain self-shielded cross sections calculated with the BONAMI module of SCAMPI at the operative temperatures and material composition background cross sections, typical of PWR and BWR material mixtures. These cross sections were collapsed with MALOCS using problemdependent neutron and photon spectra, previously calculated with XSDRNPM for the specific reactor mixtures of materials. The required weighting flux spectra were obtained from two XSDRNPM calculations simulating the simplified in-vessel and ex-vessel one-dimensional radial geometries, typical of a PWR and a BWR. The spectrum calculations were performed with finegroup self-shielded cross sections, separately derived from VITJEFF311.BOLIB and VITENDF70.BOLIB. Concerning the six blocks of self-shielded cross sections in each library, there are in particular: 1) a block of PWR core cross sections collapsed on a PWR core spectrum, 2) a block of cross sections for coolant materials collapsed on a PWR downcomer spectrum, 3) a block of cross sections for PWR pressure vessel materials collapsed on a PWR pressure vessel spectrum at the one quarter thickness position (1/4 T) in the pressure vessel, 4) a block of cross sections for concrete materials of a reactor biological shield collapsed on a concrete PWR biological shield spectrum, 5) a block of carbon and stainless steel materials respectively for the LWR pressure vessel and internals and finally, 6) a block of cross sections for BWR core materials collapsed on a BWR core spectrum. The NITAWL and ALPO modules of SCAMPI were used for format conversion, respectively from the AMPX master to the AMPX working format and from the AMPX working to the ANISN format while the RADE module of SCAMPI was used to perform data consistency tests. It is underlined that the U-235, U-238 and Pu-239 fission spectra contained in BUGJEFF311.BOLIB and BUGENDF70.BOLIB, differently from those included in BUGLE-96 which contain only the corresponding prompt neutron spectra, are total (prompt + delayed) neutron fission spectra obtained simply summing up, in each neutron broad-group, the corresponding fine-group data included in VITJEFF311.BOLIB and VITENDF70.BOLIB. BUGJEFF311.BOLIB and BUGENDF70.BOLIB contain response functions [22] for all the 71 threshold dosimeter cross sections, directly obtained from the IAEA International Reactor Dosimetry File 2002 [23] (IRDF-2002). The GROUPIE module of the PREPRO-2007 [24] nuclear data processing system was used to perform the data processing of the point-wise dosimeter cross section files at the temperature of $300{ }^{\circ} \mathrm{K}$ into the BUGLE-96 neutron group structure. These response functions are available in each library package with two flux weightings: flat flux spectrum and 1/4 T PWR pressure vessel spectrum. In order to obtain the response functions with the second weighting, the corresponding fine-group spectra, separately calculated with XSDRNPM from VITJEFF311.BOLIB and VITENDF70.BOLIB, were 
used. All the geometrical, compositional and temperature data needed for the calculations of the PWR and BWR neutron and photon spectra and for the generation of the BUGJEFF311.BOLIB and BUGENDF70.BOLIB self-shielded cross section blocks were consistently taken from the corresponding ORNL inputs of SCAMPI which generated BUGLE-96. Moreover these libraries are available also with the thermal upscattering cross sections retained in the thermal neutron energy region below about $5.0 \mathrm{eV}$ and these versions are named BUGJEFF311T.BOLIB and BUGENDF70T.BOLIB. BUGJEFF311.BOLIB and BUGENDF70.BOLIB were preliminarily tested using the TORT-3.2 $3 \mathrm{D} \mathrm{S}_{\mathrm{N}}$ code on the PCA-Replica $12 / 13\left(\mathrm{H}_{2} \mathrm{O} / \mathrm{Fe}\right)$ and VENUS-3 low-flux engineering neutron shielding benchmark experiments (SINBAD [25] database), specifically dedicated to improve the accuracy of the neutron fluence calculations in PWR structural components. Concerning PCA-Replica, an experimental array including alternate regions of water and steel with a PWR pressure vessel simulator, the calculated reaction rates for the Rh-103(n,n')Rh$103 \mathrm{~m}$, In-115(n,n')In-115m and S-32(n,p)P-32 threshold detectors, obtained with flat weighting dosimeter cross sections, are within the desired target accuracy of $\pm 10 \%$ with respect to the experimental data. With respect to VENUS-3, a reactor with a cruciform core with typical PWR fuel assemblies and PWR neutron spectrum, the calculated results of both libraries for the 386 In115(n,n')In-115m, Ni-58(n,p)Co-58 and Al-27(n, $\alpha)$ Na-24 threshold detectors, in terms of equivalent fission fluxes, are within the desired target accuracy of $\pm 10 \%$ with respect to the experimental data.

\section{Conclusion}

Is is expected that the present four ENEA-Bologna libraries can contribute to the promotion of the use of the deterministic codes in shielding and radiation damage calculations in nuclear fission reactors. Further validation is foreseen for the broad-group working libraries.

\section{References}

1. W.A. Rhoades, D.B. Simpson, TORT Version 3, ORNL/TM-13221 (October 1997).

2. R. Orsi, ENEA FPN-P9H6-011 (October 2008).

3. R. Orsi, Nuc. Sci. and Eng., Tech. Note, 146, pp. 248-255 (2004).

4. Y. Hirao, K. Ahiko, J. of Nuc. Sci. and Tech., 47, No. 2, pp. 127-135 (2010).

5. DOORS3.1, ORNL, RSIC CCC-650 (August 1996).

6. Am. Nat. Standard, ANSI/ANS-6.1.2-1999 (R2009) (2009).

7. J.E. White et al., ORNL-6795/R1, NUREG/CR-6214, Revision 1 (January 1995).

8. I.I. Bondarenko et al., Group constants for nuclear reactors calculations (Cons. Bureau 1964).

9. The JEFF-3.1.1 Nuclear Data Library, JEFF Report 22, OECD-NEA Data Bank (2009).

10. The JEFF-3.1 Nuclear Data Library, JEFF Report 21, OECD-NEA Data Bank (2006).

11. M.B. Chadwick et al., Nuc. Data Sheets, 107, 12, pp. 2931-3060, (December 2006).

12. M. Pescarini, V. Sinitsa, R. Orsi, ENEA UTFISSM-P9H6-003, (November 2011).

13. M. Pescarini, V. Sinitsa, R. Orsi, M. Frisoni, ENEA UTFISSM-P9H6-005 (May 2012).

14. R.E. MacFarlane, NJOY-99, "README0", ORNL, RSIC PSR-0480/02 (December 1999).

15. V. Sinitsa, M. Pescarini, ENEA FPN-P9H6-006 (September 2007).

16. SCAMPI, ORNL, RSIC PSR-352 (September 1995).

17. J.M. Risner et al., ORNL/TM-2011/12, NUREG/CR-7045 (September 2011).

18. R.W. Roussin et al., VITAMIN-C, ORNL/RSIC-37 (1980).

19. ICSBEP, OECD-NEA NSC, NEA/NSC/DOC(95)03 (September 2004 Edition).

20. M. Pescarini, V. Sinitsa, R. Orsi, M. Frisoni, ENEA UTFISSM-P9H6-002 (May 2011).

21. M. Pescarini, V. Sinitsa, R. Orsi, M. Frisoni, ENEA UTFISSM-P9H6 report to be published.

22. R. Orsi, M. Pescarini, V. Sinitsa, ENEA UTFISSM-P9H6-006 (May 2012).

23. O. Bersillon et al., IRDF-2002, IAEA, Tech. Reports Series No. 452 (2006).

24. D.E. Cullen, PREPRO 2007, LLNL, IAEA-NDS-39, Rev. 13 (March 2007).

25. SINBAD, OECD-NEADB/ORNL-RSICC, SINBAD REACTOR, NEA-1517 (2009 Edition). 